Publicación semestral. ISSN 2215-4906

Volumen 81 - Número 1 Julio - Diciembre 2021

\title{
La investigación-creación en diálogo con la escena teatral digital
} Research-creation in Dialogue
with Digital Theater

Paula Rojas Amador

DOI 10.15517/es.v81i1.47288

(c) (i) (2) Esta obra está bajo una licencia Creative Commons
BY NG ND Reconocimiento-No comercial-Sin Obra Derivada 
Reflexiones

\title{
La investigación-creación en diálogo con la escena teatral digital

\author{
Research-creation in Dialogue \\ with Digital Theater
}

\author{
Paula Rojas Amador ${ }^{1}$ \\ Universidad Nacional de Costa Rica \\ Heredia, Costa Rica
}

Recibido: 5 de marzo del 2020 Aprobado: 10 de febrero de 2021

\section{¿A qué nos referimos al hablar de investigación-creación?}

La respuesta a ¿qué es una investigación-creación? se convierte en un elemento esencial para todo investigador-creador al partir del hecho de que su rol cambia, desde el primer momento, al de un practicante reflexivo (Bruneau \& Burns, 2007). Además, la investigación-creación implica el cuestionamiento y la confrontación con nuestras propias estructuras de pensamiento disciplinares, y una mirada crítica a las experiencias investigativas basadas en esta dualidad y en la oposición de los paradigmas cuantitativo-cualitativo.

El presente texto comparte y discute, en un primer momento, la visión de autores como: Monique Bruneau y Sophia L. Burns (2007), Robin Nelson (2013), Brad Haseman (2006), quienes postulan la investigación-creación como un enfoque que implica un venir e ir constante entre la teoría y la práctica y una escucha de las características propias de cada objeto-sujeto de estudio, por lo tanto, la metodología es singularizada a cada trabajo. Además, en este enfoque es central la rigurosidad de la sistematización de los insumos provenientes de la práctica para producir y crear el diálogo e intercambio esperado con la teoría. En un segundo momento, este trabajo busca analizar el por qué la investigación-creación

1 Profesora, académica, investigadora-creadora de la Escuela de Arte Escénico de la Universidad Nacional de Costa Rica. Ph.D. en Literatura, Artes Escénicas y Cinematográficas por la Universidad Laval de Québec-Canadá. ORCID: 0000-0001-7563-361X. Correo electrónico: paula.rojas.amador@una.cr 
es uno de los enfoques más apropiados para las investigaciones que abordan la relación entre arte y tecnología.

De acuerdo con Izabella Pluta y Mireille Losco-Lena (2015, pp.47-58), la práctica de la investigación-creación es bastante común en Francia, Australia, Canadá, Reino Unido y en los países nórdicos. Aunque hay diferencias de un país a otro, las autoras señalan la consolidación y la institucionalización de la investigación-creación que han tenido lugar desde hace mucho tiempo en Canadá y en el Reino Unido. De la misma manera, es importante señalar la proliferación de este enfoque en países como Brasil, Chile, Colombia y México. Una de las definiciones propuestas de investigación-creación es la de Sophie Stévance y Serge Lacasse, quienes indican que:

La recherche-création consiste en la mise en place d'espaces de dialogue et d'échange, sur la base de stratégies discursives appliquées, entre la recherche scientifique et la création artistique. Un projet de recherche-création correspond donc à une démarche de recherche établie à partir d'un processus de création ou à travers celui-ci, encourageant dans son sillon la diffusion double d'une production artistique et d'un discours de nature théorique [La investigación-creación consiste en la puesta en marcha de los espacios de diálogo y de intercambio, sobre la base de estrategias discursivas aplicadas, entre la investigación científica y la investigación artística. Un proyecto de investigación-creación corresponde así a un proceso de investigación establecido a partir de un proceso de creación o a través de él, fomentando a su vez la doble difusión de una producción artística y un discurso de naturaleza teórica] (2013, p. 122).

Así, la investigación-creación surge de un ir y venir reflexivo entre la construcción teórica y la creación artística, entre el concepto y la experimentación. Al respecto, Monik Bruneau y Sophia L. Burns escriben: "en recherche-création, la création est l'enjeu même de sa recherche et le praticien ne peut résoudre son énigme sans plonger dans sa propre pratique. L'enjeu varie: comprendre, innover, transformer une pratique dans son sens large [en investigación-creación, la creación es la apuesta misma de la investigación y el practicante no puede resolver su enigma sin sumergirse en su propia práctica. La apuesta varía: comprender, innovar, transformar una práctica en un sentido amplio]" (2007, p. 172). Al visualizar la creación como objeto y sujeto de la investigación a la vez, este enfoque implica una experiencia de creación/teorización que no es evidente y, por lo tanto, el cómo llevarla a cabo requiere claridad sobre lo que se quiere profundizar de la práctica. 
En cuando al vínculo entre el arte y la tecnología, y su indisoluble relación entre el objeto y sujeto, podemos visualizarlo con el siguiente ejemplo: el investigador-creador, Émile Beauchemin, se propone a desarrollar lo que él denomina Interface Humaine ${ }^{2}$. Este consiste en la construcción de un dispositivo que es adherido a su cuerpo y a través del cual controla la luz, el video, el sonido y los sensores en tiempo real en la escena, mientras interpreta un monólogo de Hamlet.

\section{Posibles motivaciones al hacer una investigación-creación}

Las razones que llevan a un artista a realizar una investigación-creación son diversas. Sabemos que existe un conocimiento tácito, implícito, silencioso que surge en los procesos artísticos y de la manera de crear, de pensar, de hacer y visualizar el arte que está únicamente en el mismo artista. Es este quien puede dialogar, activar, descubrir, o bien redescubrir las razones y los motivos que lo llevan a crear. El instinto, la experiencia, las técnicas empleadas, los trazos de sus obras y los deseos artísticos que lo transforman y que lo hacen cuestionarse a sí mismo. Este saber puede ser compartido, puesto en diálogo con otros conceptos y otras prácticas, los cuales, sumados a la sistematización y reflexión, producen un conocimiento que, inevitablemente, traspasa y requiere de la participación activa del artista-investigador.

De esta manera, la persona artista puede tomar la decisión de realizar una investigación-creación por el deseo de aumentar la efectividad interpretativa, poética y/o estética de su trabajo; puede tener el deseo de conocer o profundizar una técnica específica, un conocimiento determinado de su propia disciplina o querer aproximarse a un trabajo interdisciplinario, multidisciplinario o intermedial. Asimismo, podría tener interés en explorar nuevas materialidades, espacialidades, temporalidades u otros vínculos con el espectador, y ver en una investigación-creación un espacio propicio para entender su propia creación, para vitalizarla, renovarla, innovarla, o bien autodescubrir su propia obra.

Para ahondar en los diversos intereses y motivaciones que llevan a los artistas a realizar una investigación-creación, tomaremos cuatro escenarios posibles descritos por Bruneau y Burns, que nos permiten un panorama amplio de las acciones que pueden ser concretizadas según sea el caso. Los escenarios son los siguientes:

\footnotetext{
2 Véase Interface Humaine | Interférences (interferences.ca)
} 
Scenario A. Faire de sa pratique artistique un lieu et la manifestation d'un projet d'innovation, d'expérimentation (étude constructiviste, exploratoire, heuristique). Scenario B. Théoriser sa pratique par l'élaboration d'un projet artistique (poétique, auto ethnographique, phénoménologique, herméneutique...). Scenario C. Positionner sa pratique en fonction d'un cadre historique, culturel, social, selon une thématique ou une combinaison de ces données (étude critique, féministe, recherche intervention...). Scenario $D$. Réléver un phénomène par une production artistique (heuristique, descriptive, expérimentale...) [Escenario A. Hacer de su práctica artística un lugar y una manifestación de un proyecto de innovación, de experimentación (estudio constructivista, exploratorio, heurístico). Escenario B. Teorizar su práctica para la elaboración de un proyecto artístico (poético, autoetnográfico, fenomenológico, hermenéutico...). Escenario C. Posesionar su práctica en función de un cuadro histórico, cultural, social, según una temática o una combinación de estos datos (estudio crítico, feminista, investigación intervención...). Escenario D. Resaltar un fenómeno para una producción artística (heurística, descriptiva, experimental...)] (2007, pp. 165-174)

En el primer escenario, la tesis se centra en un proyecto de innovación y experimentación. Según Bruneau y Burns, en este escenario "Ia pratique artistique est le moyen (lieu d'expérimentation), l'objet de recherche, le sujet de recherche et la résultante du processus de théorisation et de création [la práctica artística es el medio (lugar de experimentación), el objeto de investigación, el sujeto de investigación y el resultado del proceso de teorización y de creación]" (2007, p. 165). Por lo que, de acuerdo con las autoras, este caso va dirigido aquellos practicantes que tienen como reto la renovación de su práctica o de la formación artística.

Asimismo, para el primer escenario se tiene por pregunta clave: ¿cómo se puede hacer esto? En vez de, ¿por qué hacerlo de cierta manera? La creación de laboratorios de exploración y experimentación son comunes en este caso, por lo que no es requisito la conclusión de una obra artística. Además, podemos observar que la presencia de la innovación y experimentación convoca a los estudios de arte y tecnología.

Vinculado a este tipo de trabajo, en el cual se busca la innovación, podemos mencionar un segundo ejemplo: la tesis de maestría titulada Recherche-création: éclairage-vidéo : le projecteur vidéo comme source d'éclairage au théâtre [La investigación-creación: 
iluminación-video: el proyector como fuente de luz en el teatro] ${ }^{3}$ de Keven Dubois (2014). Dubois se propone utilizar el proyector como fuente lumínica, pero no desde un uso tradicional cinematográfico o educativo, sino que recurre al desarrollo de diversas experimentaciones artísticas que dan cuenta del potencial y ventajas que este uso particular del proyector tiene para la escena.

El segundo escenario, "il s'agit d'une démarche d'investigation inductive qui se développe dans la pratique et par la pratique [se trata de un tipo de investigación inductiva que se desarrolla en la práctica y para la práctica]" (Bruneau \& Burns, 2007, p. 166). En este caso, es imprescindible la creación de una obra artística que permita, durante el mismo proceso de creación, comprender y explicar ciertos aspectos de su propia práctica y teorizarla. Este caso está dirigido a los artistas que desean "comprendre les fondements de leur propre pratique, certains aspects ou phénomènes occurrents, leurs influences, leur processus, leurs moments de rupture... [comprender los fundamentos de su propia práctica, ciertos aspectos o fenómenos recurrentes, sus influencias, sus procesos, sus momentos de ruptura...]" (Bruneau \& Burns, 2007, p. 166). Por tanto, para el escenario dos, no se tiene como pretensión innovar la manera de crear, sino comprenderla y teorizarla.

Ahora bien, el tercer escenario es dirigido al practicante que tiene como desafío "comprendre sa pratique en la situant au regard d'un cadre historique, social ou culturel donné sur lequel on pose ou nom un regard critique [comprender su práctica al situarla a la luz de un cuadro histórico, social o cultural específico sobre el cual se coloca o no una visión crítica]" (Bruneau \& Burns, 2007, p. 168). Este tipo de investigación-creación puede concretizarse en formatos de trabajo como historia de vida, autoetnográficos con soportes fotográficos o videográficos, por ejemplo.

Finalmente, en el cuarto escenario el investigador busca evidenciar "la puissance évocatrice de l'art, soutenant que la connaissance émerge d'une réalité construite par l'interprétation qu'on lui donne au regard du contexte auquel cette réalité appartient [el poder evocativo del arte, sosteniendo que el conocimiento emerge de una realidad construida por la interpretación que uno mismo le da al observar el contexto en el cual esta realidad pertenece]" (Bruneau \& Burns, 2007, p. 169). El practicante tiene un deseo de modificar

${ }^{3}$ Veáse: CorpusUL: Recherche-création : éclairage-vidéo : le projecteur vidéo comme source d'éclairage au théâtre (ulaval.ca). 
la manera de observar, de percibir y comprender una realidad. Además, desea indagar a través de qué tipo de procesos, de cuáles herramientas o formas, el arte revela un fenómeno social, cultural, artístico, político u otro. En este escenario, se tratan temáticas como la guerra del Golfo, el hambre, el terrorismo, la exclusión, la inmigración, por ejemplo.

Como hemos podido entrever, mediante estos escenarios, en la investigación-creación, la posición del investigador-creador se convierte en uno de los elementos clave. La claridad sobre lo que lo motiva a utilizar este enfoque es fundamental en el proceso: ¿qué desea comprender de su práctica?, ¿qué desea interrogar de su práctica?, ¿qué desea innovar de su práctica? Es importante señalar que si bien estos escenarios reúnen ciertos elementos recurrentes de la práctica en investigación-creación, los mismos no pretenden delimitarla. Recordemos que el enfoque y la metodología dependen de cada objeto/sujeto de estudio, ofreciéndonos muchos otros escenarios posibles.

\section{El practicante-reflexivo: sistematización y herramientas}

Uno de los aspectos vitales a considerar en la puesta en marcha de una investigación-creación es la rigurosidad de la recopilación de los datos, la observación y la sistematización de los procesos. Esto confiere al trabajo el carácter científico con el que dialoga este enfoque. Como lo afirma Simon Hagemann

si l'on considère la recherche comme une activité systématique qui mène à la production d'un savoir nouveau, on peut considérer la recherche-création comme une activité artistique systématique, dont le critère général est sa capacité à participer à la production d'un savoir [si consideramos la investigación como una actividad sistemática que nos lleva a la producción de un conocimiento nuevo, podemos considerar la investigación-creación como una actividad artística sistemática, donde su criterio general es su capacidad de participar en la producción de conocimiento] (2015, p. 89).

Es decir, el rol del investigador pasa a ser el de un practicante reflexivo, tal cual lo definen Monik Bruneau y Sophia L. Burns, al indicar que

le praticien réflexif, en plus d'être un praticien expérimenté, est un chercheur, c'està-dire un étudiant qui a la volonté de comprendre et d'expliquer la réalité de sa pratique. Le praticien réflexif se trouve donc à cheval sur deux logiques : celle du praticien qui croit que l'action précède le savoir et celle du chercheur qui croit que le savoir qu'il tente de retracer précède l'action. ... Le praticien réflexif devient, selon 
son engagement, un chercheur particulier conscient de la force et de la valeur de l'expérience pratique de l'artiste, du formateur en art et de la fragilité de ce savoir qui se perdront si nous ne prenons pas le temps d'analyser ces expériences, d'évaluer les effets de ces actions sur leurs résultats et sur la formation en art [el practicante reflexivo, además de ser un practicante experimentado, es un investigador, esto quiere decir un estudiante que tiene la voluntad de comprender y de explicar la realidad de su práctica. El practicante reflexivo se encuentra así a caballo sobre dos lógicas: la del practicante que cree que la acción precede su conocimiento y la del investigador que cree que el conocimiento que el intenta de trazar precede la acción. ... El practicante reflexivo se convierte, de acuerdo a su compromiso, en un investigador particular consciente de la fuerza y del valor de la experiencia práctica del artista, del formador en artes y de la fragilidad de este conocimiento que se perderá si nosotros no tomamos el tiempo de analizar estas experiencias, de evaluar los efectos de estas acciones sobre sus resultados y sobre la formación en arte] (2007, pp. 156-157).

Para un practicante reflexivo, existe el deseo de descubrir el conocimiento que puede obtener de su práctica, al adoptar una manera sistemática de ver y un anhelo de observar cómo su reflexión sobre su propia práctica se traduce en esta y viceversa. Asimismo, desarrolla los conceptos y herramientas que permiten el estudio y la evaluación de las diferentes tareas y los diferentes estados de su creación.

Una de las investigaciones-creaciones que podemos mencionar, para ejemplificar esta necesidad de sistematizar y elaborar herramientas propias para el trabajo, es la tesis de doctorado Les médias numériques dès une écriture intermédiale : suivi de Le monde n’arrête pas, scénario dramaturgique [La tecnología digital desde una escritura intermédial: seguida del guión-dramatúrgico El Mundo no para] ${ }^{4}$ (2018) de mi autoría. En esta investigación-creación se desarrollan una serie de dispositivos escénicos visuales, sonoros, espaciales y textuales dentro de un contexto de laboratorio. De este espacio emanan: maquetas digitales, contenidos audiovisuales, concepciones escenográficas, creaciones dramatúrgicas intermediales, procedimientos y métodos de trabajo, los cuales pueden ser visualizados dentro de un sitio web ${ }^{5}$ y que, en conjunto con la tesis, responden a la problemática

${ }^{4}$ Veáse: CorpusUL: Les médias numériques dès une écriture intermédiale : suivi de Le monde n'arrête pas, scénario dramaturgique (ulaval.ca).

5 Veáse: Rojas Amador (myportfolio.com). 
de la adaptación de un cuento al teatro en un contexto intermedial. El diálogo continuo entre teoría y práctica, y la rigurosa sistematización del proceso investigativo-creativo permitió, posteriormente, dar forma al Protocole de travail pour les laboratoires de recherche scénique [Protocolo de trabajo para los laboratorios de investigación escénica] (2018, pp. 142-146). Este constituye un insumo pedagógico para aquellas personas que se introducen en la escena digital.

Robin Nelson revela también particularidades en el trabajo del practicante-investigador, entre ellas: "spécifier l'enquête depuis le début, introduire des moments de réflexion critique dans le calendrier, placer sa recherche dans une relation avec d'autres recherches menées sur des sujets similaires et mettre cette recherche en lien avec les débats contemporains [especificar lo que se investiga desde el inicio, introducir los momentos de reflexión crítica en el calendario, colocar su investigación en relación con otras investigaciones sobre sujetos similares y colocar esta investigación en vínculo con los debates contemporáneos]" (citado por Hagemann, 2015, p. 89). Así, la dinámica de la investigación-creación y los desafíos que surgen provocan que este enfoque sea visto como poco tradicional en el tratamiento del sujeto. De igual manera, el corpus y la metodología de la investigación-creación requieren de un diálogo crítico para lograr exitosamente los retos que son propuestos desde esta otra posición de practicante reflexivo.

\section{Una epistemología y/o múltiples epistemologías}

Otro elemento que requiere de análisis es el enfoque epistemológico que es aplicado a este tipo de trabajo, pues el objeto de estudio en la investigación-creación se vuelve único y particular. En este caso, el trabajo puede ser abordado por una o diversas epistemologías. En particular, encontramos la metodología general híbrida propuesta por Bruneau y Burns. Según estas autoras, esta metodología

permet au chercheur de s'adapter aux contingences de l'objet qui l'intéresse, en prenant en compte le contexte et en introduisant dans son protocole de recherche des outils et des démarches appartenant à des épistémologies ou à des méthodologies différentes. Le chercheur devient le maitre d'œuvre du design méthodologique [permite al investigador adaptarse a las contingencias del objeto que le interesa, tomando en cuenta el contexto e introduciendo en su protocolo de investigación herramientas y procedimientos pertenecientes a otras epistemologías y metodologías diferentes. El investigador se convierte en el maestro de obra de su diseño metodológico] (2007, p. 84). 
Otro modelo interesante para abordar una investigación-creación es el de modos de conocimiento: modelo epistemológico multi-modo para la práctica como investigación, presentado por Robin Nelson en su libro Practice as research in the art: Principles, protocols, pedagogies, resistances [Práctica como investigación en artes: principios, protocolos, pedagogías, resistencias] (Nelson, 2013, pp. 37-47). En este modelo, la práctica en artes entrelazada con la teoría es el núcleo. Para el autor, la clave de este enfoquees aceptar "that knowledge is not fixed and absolute [que el conocimiento no es fijo y absoluto]" (2013, p. 39).

Asimismo, en este modelo se consideran tres grandes elementos. Primero, el saber hacer "know-how", que se refiere a las experiencias y conocimiento artístico y tácito del investigador-creador, que lo han llevado a este planteamiento. Después, el saber qué "knowwhat", el cual se centra en los conceptos, las palabras, los procedimientos y los análisis reflexivos que surgen cuando la investigación-creación está en proceso de realizarse, lo que puede también ser llamado haciendo-conociendo "doing-knowing" o reflexión en acción "reflects-in-action" (2013, p. 65). Y finalmente, el saber que "know-that" que nos envía al cuadro conceptual y las teorías que permiten un diálogo con la práctica.

En lo que concierne a la epistemología del proyecto, la investigación-creación se construye según sus necesidades específicas. Nelson afirma que "a key observation to reiterate in respect of my epistemological model, then, is that the whole dynamic and interactive [una observación clave a recordar a propósito de este modelo epistemológico es que el conjunto es dinámico e interactivo]" (2013, p. 62). Dicho de otra manera, hay una constante influencia entre el saber hacer "know-how", el conocimiento que surge de la práctica "knowwhat" y el saber que "know-that".

Ahora bien, este modelo propone que "theory, that is to say, is not prior to practice, functioning to inform it, but theory and practice are rather 'imbricated within each other' in praxis [la teoría, no es anterior a la práctica, sirve para informarla, pero la teoría y la práctica están más bien 'estrechamente unidas una dentro de la otra']" (2013, p. 62). Por tanto, la praxis hace igualmente una llamada a la subjetividad y a la intuición que forman parte de todo el proceso investigativo-creativo.

Finalmente, otro enfoque de esta investigación-creación es la propuesta de Brad Haseman en su texto A Manifesto for Performative Research [El manifiesto para la investigación performativa]. El autor propone un paradigma performativo, el cual no está guiado por números -referencia al paradigma cuantitativo- ni por palabras -relación con el paradigma 
cualitativo-, sino por un lenguaje y forma que corresponda a su práctica. Por ejemplo, el escritor a través de la novela, el cuento y la literatura construye su lenguaje y forma de investigar. Lo mismo sucede con el compositor y su música, con el bailarín y la coreografía, o con el actor a través de la interpretación. Paradigmas que podemos observar de manera resumida-comparativa en el siguiente cuadro:

Cuadro 1. Paradigmas de investigación, cuadro comparativo

\begin{tabular}{|c|c|c|}
$\begin{array}{c}\text { Quantitative Research } \\
\text { [Investigación cuantitativa] }\end{array}$ & $\begin{array}{c}\text { Qualitative Research } \\
\text { [Investigación cualitativa] }\end{array}$ & $\begin{array}{c}\text { Performative Research } \\
\text { [Investigación performativa] }\end{array}$ \\
\hline $\begin{array}{l}\text { The activity or operation of } \\
\text { expressing something as a } \\
\text { quantity or amount - for } \\
\text { example, in numbers, graphs, } \\
\text { or formulas [La actividad u } \\
\text { operación de expresar algo } \\
\text { como una cantidad o porción - } \\
\text { Por ejemplo, en números, } \\
\text { gráficos o fórmulas] } \\
\text { (Schwandt, 2001, p. 215). }\end{array}$ & $\begin{array}{l}\text { Refers to 'all forms of } \\
\text { socialinquiry that rely } \\
\text { primarilyon qualitative } \\
\text { data...i.e., nonnumeric data } \\
\text { in the form of words' } \\
\text { [Se refiere a 'todas } \\
\text { las formas de investigación } \\
\text { social que se basan } \\
\text { principalmente en datos } \\
\text { cualitativos... Esto es, los } \\
\text { datos no numéricos en } \\
\text { forma de palabras'] } \\
\text { (Schwandt, 2001, p. 213). }\end{array}$ & $\begin{array}{l}\text { Expressed in nonnumeric } \\
\text { data, but in forms of } \\
\text { symbolic data other than } \\
\text { words in discursive text. } \\
\text { These include material } \\
\text { forms of practice, of still } \\
\text { and moving images, of music } \\
\text { and sound, of live action and } \\
\text { digital code [Expresada en } \\
\text { datos no numéricos, en forma } \\
\text { de datos simbólicos diferentes } \\
\text { de palabras de un texto } \\
\text { discursivo. Estos incluyen } \\
\text { formas materiales de práctica, } \\
\text { de imágenes fijas y en } \\
\text { movimiento, de música y del } \\
\text { sonido, de acción en vivo } \\
\text { código digital. }\end{array}$ \\
\hline $\begin{array}{l}\text { The scientific method } \\
\text { [El método científico] }\end{array}$ & $\begin{array}{l}\text { Multi-method } \\
\text { [Multi-método] }\end{array}$ & $\begin{array}{l}\text { Multi-method led by practice } \\
\text { [Multi-método guiado } \\
\text { por la práctica] }\end{array}$ \\
\hline
\end{tabular}

Fuente: Haseman, 2006, p. 7.

Es posible observar que en la metodología híbrida de Bruneau y Burns; en el modelo de práctica como investigación de Nelson, y en el paradigma performativo de Haseman se comparte el hecho que la epistemología debe estar a la escucha de las características propias de cada investigación-creación. Lo anterior, sin perder de vista esta tensión dinámica entre teoría y práctica, la cual es esencial para el desarrollo del conocimiento en este abordaje investigativo-creativo.

ESCENA. Revista de las artes, 2021, Vol. 81, Núm. 1 (julio-diciembre), pp. 495-508 
Como hemos visto en los ejemplos brindados, se trata de trabajos que se trasladan constantemente entre diversas disciplinas: ingeniería, audiovisual, robótica, programación e informática, de las cuales el teatro figura entre ellas. Esta realidad evidencia por qué su diseño metodológico varía según el objeto-sujeto de estudio. Una vez que hemos planteado, brevemente, lo que es la investigación-creación, las diversas motivaciones que llevan a un artista a realizar este tipo de trabajo, el rol del practicante-reflexivo y los abordajes epistemológicos posibles, nuestro interés es analizar el por qué la investigación-creación es uno de los enfoques más apropiados para las indagaciones que involucran la relación arte y tecnología.

\section{Investigación-creación en procesos artísticos vinculados al arte y la tecnología}

Como hemos visto, la perspectiva propuesta por la investigación-creación dialoga con procesos investigativos que buscan innovación, creación de dispositivos escénicos, formas de hacer arte con técnicas, materiales, así como metodologías novedosas y originales. Cuando se trata de investigaciones-creaciones que involucran la tecnología, se incurre en la necesidad de espacios de exploración, experimentación, creación de prototipos; equipos de trabajo inter, multi y transdisciplinares, y la sistematización de esta experiencia entre el arte y la tecnología. En dichos procesos es frecuente la aparición de laboratorios de creación para llevar a cabo estos procesos investigativos.

En el teatro, podemos referirnos a creadores como Constantin Stanislavski (18631938), Vsévold Meyerhold (1874-1940) y Jerzy Grotowski (1933-1999), quienes propiciaron espacios de exploración y experimentación sobre la técnica actoral y cuyos aportes son sumamente valiosos en esta área. Tenemos que tomar en cuenta que para los trabajos artísticos que implican una práctica con la tecnología digital, es común la utilización de imágenes, de sonidos, de hologramas, de personajes virtuales, los cuales requieren ser puestos a prueba, explorados y reflexionados en un espacio práctico, como los laboratorios, para que su efectividad en la escena o en la creación pueda ser garantizada. Si bien emerge en el contexto de la investigación-creación, la figura del laboratorio en el arte, también surgen nuevos perfiles como artistas-ingenieros o artistas de laboratorio. Perfiles que exigen a los artistas otros conocimientos, distintos o complementarios al arte, lo cual les permite interactuar con el mundo virtual de una manera más directa en la escena.

Cuando trabajamos en una investigación-creación que incluye la tecnología nace una obra artística, pero también surgen procedimientos, dispositivos escénicos y sitios web que se convierten en valores agregados al trabajo y, consecuentemente, también tienen un valor académico y de difusión del trabajo. Al respecto, Jean-Paul Fourmentraux afirma que 
la valorisation d'une recherche-création en art et technologie devra ainsi favoriser l'émergence d'une œuvre et en faire simultanément un "milieu" producteur d'innovations technologiques : l'œuvre peut y être multiple, intermédiaire et fragmentaire, la recherche qui y mène pourra également être valorisée (procédés, méthodes, connaissances) et parfois donner lieu à des productions autres que l'œuvre visée (outils, logiciels, dispositifs scéniques, etc.) [la valorización de una investigación-creación en arte y tecnología favorecerá la aparición de una obra y, al mismo tiempo, la convertirá en un "medio" que produce innovaciones tecnológicas: la obra puede ser múltiple, intermedia y fragmentaria, la investigación por sí también puede ser valorada (procesos, métodos, conocimientos) y, a veces, dar lugar a producciones distintas del trabajo previsto (herramientas, software, dispositivos escénicos, etc.)] (2011, p. 35).

La investigación-creación en arte y tecnología lleva al desarrollo de competencias, las cuales un creador teatral no hubiera pensado requerir y que le permiten construir desde animaciones de marionetas digitales, secuencias de sonido e imagen, hasta captores, lentes 3D y prototipos. Esto conduce a los artistas a la implementación de laboratorios escénicos, investigaciones teórico-práctica y a modelos de investigación como el de investigación-creación sobre el cual hemos conversado.

Para cerrar, si la investigación-creación surge lentamente en el contexto universitario de ciertos países; en otros, es una práctica cotidiana. Podemos notar que este tipo de investigación es apoyado por diversos autores del mundo, dada la progresión de la cultura digital. Principalmente, cuando nos referimos a la incorporación de la tecnología digital en la escena, es posible afirmar que la investigación-creación se presenta como uno de los modelos más apropiados para abordar este tipo de experiencias en la escena tecnológica.

\section{Referencias}

Bruneau, M. \& Burns, S. (2007). Se faire praticien réflexif : tracer une route de recherche en art. En el Traiter de recherche création en art. Quebec, Canadá: Editorial de la Universidad de Quebec.

Dubois, K. (2014). Recherche-création : éclairage-vidéo : le projecteur vidéo comme source d'éclairage au théâtre. (Tesis de maestría en Littérature et arts de la scène et de l'écran). Universidad de Laval, Quebec, Canadá. 
Fourmentraux, J. (2011). Artistes de laboratoire : recherche et création à l'ère numérique. París: Hermann.

Hagemann, S. (2015). La recherche des avant-gardes théâtrales historiques autour du progrès technique. Ligeia, 28(137-140), 88-96.

Haseman, B. (2006). A Manifesto for Performative Research. Media International Australia incorporating Culture and Policy, (118), 98-106.

Nelson, R. (2013). Practice as research in the art: Principles, protocols, pedagogies, resistances. London: Palgrave Macmillan.

Pluta, I. \& Mireille L. (2015). Pour une topographie de la recherche-création. Ligeia, 28(137140), 47-58.

Rojas, P. (2018). Les médias numériques dès une écriture intermédiate suivi de Le monde n'arrête pas, scénario dramaturgique. (Tesis doctoral en Littérature et arts de la scène et de l'écran). Universidad de Laval, Quebec, Canadá.

Stévance, S. \& Lacasse S. (2013). Les enjeux de la recherche-création en musique. Quebec, Canadá: Editorial de la Universidad de Quebec. 